\title{
A silkworm infection model to investigate Vibrio vulnificus virulence genes
}

\author{
MAI YAMAMOTO $^{1}$, TAKASHIGE KASHIMOTO ${ }^{2}$, YUKIHIRO YOSHIMURA ${ }^{1}$, NAO TACHIBANA ${ }^{1}$, \\ SHIHO KURODA $^{1}$, YOSHIKO MIKI ${ }^{1}$, SOU KITABAYASHI ${ }^{1}$, PING TONG ${ }^{1,3}$, JIANBO XIAO ${ }^{1,4}$, \\ KOICHI TANAKA ${ }^{1}$, HIROSHI HAMAMOTO ${ }^{5}$, KAZUHISA SEKIMIZU ${ }^{5}$ and KOICHIRO YAMAMOTO ${ }^{1}$ \\ ${ }^{1}$ Department of Nutritional Science, Okayama Prefectural University, Soja, Okayama 719-1197; \\ ${ }^{2}$ School of Veterinary Medicine, Kitasato University, Towada, Aomori 034-8628, Japan; \\ ${ }^{3}$ State Key Laboratory of Food Science and Technology, Nanchang University, Nanchang, Jiangxi 330047; \\ ${ }^{4}$ Institute of Chinese Medical Sciences, University of Macau, Taipa, Macau 0000, P.R. China; \\ ${ }^{5}$ Graduate School of Pharmaceutical Sciences, The University of Tokyo, \\ Bunkyo-ku, Tokyo 113-0033, Japan
}

Received September 2, 2015; Accepted August 26, 2016

DOI: $10.3892 / \mathrm{mmr} .2016 .5782$

\begin{abstract}
The halophilic marine bacterium, Vibrio vulnificus, occasionally causes fatal septicemia in immunocompromised patients. Mice are commonly used as experimental animals to investigate the virulence of $V$. vulnificus, however, a large number of mice are generally required for bioassays. The present study examined whether the invertebrate species, silkworms, can be used instead of mice to investigate $V$. vulnificus virulence. When the silkworms were inoculated with $1.2 \times 10^{7}$ colony forming units of $V$. vulnificus OPU1-Rf, a virulent strain of $V$. vulnificus, all injected silkworms died within $48 \mathrm{~h}$, however, those injected with culture filtrate or diluent did not. This silkworm infection model was then used to isolate attenuated $V$. vulnificus mutants from 1,016 transposon-inserted mutants. Consequently, a harmless mutant, SW998, was isolated. In this strain, the transposon was inserted into the rtxA gene, which is a known $V$. vulnificus virulence gene. In conclusion, the present study demonstrated that silkworms are useful animals for investigating the virulence of $V$. vulnificus.
\end{abstract}

\section{Introduction}

Vibrio vulnificus is a Gram-negative, comma-shaped bacterium found in warm seawater and brackish waters. $V$. vulnificus occasionally causes primary septicemia, gastroenteritis

Correspondence to: Dr Koichi Tanaka, Department of Nutritional Science, Okayama Prefectural University, 111 Kuboki, Soja, Okayama 719-1197, Japan

E-mail:ktanaka@fhw.oka-pu.ac.jp

Key words: Vibrio vulnificus, virulence, silkworm, screening, animal model and wound infections in humans. Primary septicemia and gastroenteritis can occur following ingestion of uncooked seafood contaminated with $V$. vulnificus (1), whereas wound infections are often caused by direct contact of an open wound with seawater during marine activities or in the processing of seafood (2). V. vulnificus rarely infects healthy individuals, however, immunocompromised patients with an underlying illness, including hepatic disorder, diabetes or immunodeficiency, are susceptible to infection by this bacterium (3). Among the aforementioned clinical states of $V$. vulnificus infection, primary septicemia is the most life-threatening, with a mean mortality rate of $>50 \%$ worldwide (4). There is no official national system for surveying $V$. vulnificus infections in Japan, however, it was reported that 117 individuals succumbed to mortality between 1975 and 2005 (5), and the annual number of cases of $V$. vulnificus-septicemia has been estimated to be $>200$ (6). Cases of $V$. vulnificus infection have also been reported annually in other countries. The Centers for Disease Control (Atlanta, GA, USA) reported 32 cases of $V$. vulnificus infection-associated mortality in the US in 2012 (7). In Europe, $V$. vulnificus is a known fish pathogen, particularly in eels, and $V$. vulnificus infection in humans is relatively rare (8). However, due to the global warming, there is concern of an increasing number of cases of $V$. vulnificus infection as this bacterium grows rapidly in warm seawater (9).

Several studies have reported on $V$. vulnificus virulence genes (10-12), however, the mechanism underlying the infection remains to be fully elucidated. Mice have been used as experimental animals to investigate the host-pathogen interactions of $V$. vulnificus infections, however, using a large number of mammals is expensive and raises ethical considerations $(13,14)$.

Previously, invertebrate infection models using nematodes, including Caenorhabditis elegans (15), and insects, including Galleria mellonella (16), have being applied to investigate bacterial virulence genes (17). In addition, an infection model using silkworms (Bombyx mori) for the investigation 
of bacterial pathogenesis has been reported (18). Silkworms are relatively easy to breed in laboratories, and the animal is sufficient large enough for the injection of samples into the hemolymph. The objective of the present study was to use a silkworm model to investigate the virulence of $V$. vulnificus. This model may be aid in the investigation of $V$. vulnificus virulence factors and may assist in the development of effective therapies to protect against $V$. vulnificus infection

\section{Materials and methods}

Bacterial strains, plasmids and medium. The $V$. vulnificus OPU1 strain was clinically isolated, and its rifampicin (Rf)-resistant variant, $V$. vulnificus OPU1-Rf, was used in the present study. Escherichia coli BW19795 was provided by Dr Barry L. Wanner (Purdue University, West Lafayette, IN, USA (19), which was used as a pUT donor for conjugation. E. coli $\mathrm{DH}_{10 \mathrm{~B}^{\mathrm{TM}}}$-competent cells were purchased from Life Technologies; Thermo Fisher Scientific, Inc. (Waltham, MA, USA). The signature-tagged mini-Tn $5 \mathrm{Km} 2$-y 67 transposon in the pUT delivery suicide plasmid pool was provided by Dr David W. Holden (Imperial College London, London, UK) (20). Bacterial cells were grown at $37^{\circ} \mathrm{C}$ in Luria-Bertani (LB) medium containing $10 \mathrm{~g}$ tryptone (BD Biosciences, Tokyo, Japan), $5 \mathrm{~g}$ yeast extract (BD Biosciences) and $10 \mathrm{~g}$ $\mathrm{NaCl} / \mathrm{l}(21)$, unless otherwise described. M9 minimal medium without glucose was prepared as previously described (21) and used for bacterial conjugation. The following antibiotics were added to the medium at the indicated concentrations: Rf (100 $\mu \mathrm{g} / \mathrm{ml}$; Wako Pure Chemical Industries, Ltd., Osaka, Japan), kanamycin (Km; $50 \mu \mathrm{g} / \mathrm{ml}$; Meiji Seika Pharma Co., Ltd., Tokyo, Japan) and ampicillin (Am; $100 \mu \mathrm{g} / \mathrm{ml}$; Meiji Seika Pharma Co., Ltd.). Bacterial growth was monitored by turbidity using a spectrophotometer (Spectronic 20A; Shimadzu Corporation, Kyoto, Japan).

Silkworm lethality assay. The bacteria were inoculated into silkworms using the protocol described by Hamamoto et al (22). The silkworms were raised from fertilized silkworm eggs (Hu-Yo x Tsukuba'Ne) purchased from Ehime Sansyu Co., Ltd. (Yawatahama, Japan). The eggs were incubated in a clean bench (CCV-800E-AG; Hitachi Koki Co., Ltd., Tokyo, Japan) at $25^{\circ} \mathrm{C}$ in the dark for 3-5 days, according to the manufacturer's protocol. The hatched larvae were maintained at $27^{\circ} \mathrm{C}$, humidity 60-90\% and fed artificial food (Silkmate 2S; Nosan Corporation, Yokohama, Japan) for $\sim 3$ weeks. The larvae shed their shells four times, and the fifth-instar larvae were fed antibiotic-free artificial food (Silkmate; Katakura Industries, Tokyo, Japan) for 24-26 h prior to inoculation.

The bacteria were cultivated at $37^{\circ} \mathrm{C}$ in $2 \mathrm{ml}$ of $\mathrm{LB}$ medium until the optical density at $600 \mathrm{~nm}\left(\mathrm{OD}_{600}\right)$ reached 1.0. The bacterial cultures were diluted with $10 \mathrm{mM}$ phosphate-buffered saline ( $\mathrm{pH} 7.3)$, containing $0.01 \%$ gelatin (PBSG) to cell densities of $2.4 \times 10^{7}$ and $2.4 \times 10^{8} \mathrm{cfu} / \mathrm{ml}$, and were maintained for up to $2 \mathrm{~h}$ at room temperature until they were inoculated into the silkworms. The culture filtrates were prepared by filtering the diluted cultures through a $0.45-\mu \mathrm{m}$ filter (Merck Millipore, Tokyo, Japan). A 50- $\mu 1$ aliquot of the diluted bacterial cell suspension or culture filtrate was injected into the hemolymph of the silkworms using a 1-ml plastic disposable tuberculin syringe attached to a 27-gauge needle (Terumo Corporation, Tokyo, Japan). The quantities of bacteria injected were estimated by colony forming units (cfu), determined by inoculation of the diluted bacterial culture suspensions onto LB agar and counting the number of colonies grown following incubation for $18 \mathrm{~h}$ at $37^{\circ} \mathrm{C}$. The inoculated silkworms were maintained in plastic containers without feeding, and the larval status (dead or alive) was assessed. The silkworms were considered to be dead when they showed no reaction to touch.

Construction of the transposon insertion mutants by conjugation. The transposon insertion mutants were constructed by conjugation, as described previously (23). Briefly, V. vulnificus OPU1-Rf was mated with E. coli BW19795 harboring the pUTy69 conjugative suicide plasmid, which contained the mini-Tn5Km2-y67 Km-resistant transposon. The transposon-inserted mutants were assessed by their growth on $\mathrm{Km}$ - and Rf-containing agar medium.

Cloning and sequence analysis of the attenuated mutant. The locations of the transposon-inserted regions in the mutant genome were determined by sequencing of the DNA sequence adjacent to the insertion site, as described previously (23). Briefly, whole genome DNA of attenuated mutants were extracted and digested with the restriction enzyme SalI (Nippon Gene Co., Ltd, Tokyo, Japan). The enzyme digested DNA fragments were ligated into pUC18, followed by transformation into E. coli DH10B (Thermo Fisher Scientific, Inc.). The colonies resistant to both $\mathrm{Km}$ and $\mathrm{Am}$ were selected and the plasmid DNA that ligated with the fragments containing transposon insertion sites of genomic DNA was extracted. The transposon insertion sites were determined by DNA sequencing using the primer P279 (5'-CTAGGTACC TACAACCTC-3') which anneal to the transposon. DNA sequencing was performed with an Applied biosystems DNA sequencing system and the BigDye terminator cycle sequencing kit (Thermo Fisher Scientific, Inc.). Sequence homologies were searched with the BLAST search algorithm at the National Center for Biotechnology Information (www.ncbi.nlm.nih.gov/)

\section{Results}

Lethality of V. vulnificus towards silkworms. To investigate the applicability of silkworms for in vivo $V$. vulnificus infection experiments, the present study first examined the lethality of V. vulnificus towards silkworms. The spontaneous Rf-resistant $V$. vulnificus OPU1-Rf was selected for injection into silkworms, as the infectivity and lethality of this strain have been confirmed in mice, and a mutagenesis method using the mini-Tn $5 \mathrm{Km} 2$ transposon was established (23).

Fresh V. vulnificus OPU1-Rf cultures with an $\mathrm{OD}_{600}$ of 1.0 were diluted with PBSG solution to cell densities of $2.4 \times 10^{7}$ and $2.4 \times 10^{8} \mathrm{cfu} / \mathrm{ml}$, and were maintained for up to $2 \mathrm{~h}$ at room temperature until they were inoculated into the silkworms. A 50- $\mu l$ aliquot of the diluted culture specimen was injected into the hemolymph of the silkworms from a syringe through the dorsal surface, in a manner similar to that of infection into the human blood stream. The movement of the silkworms reduced 


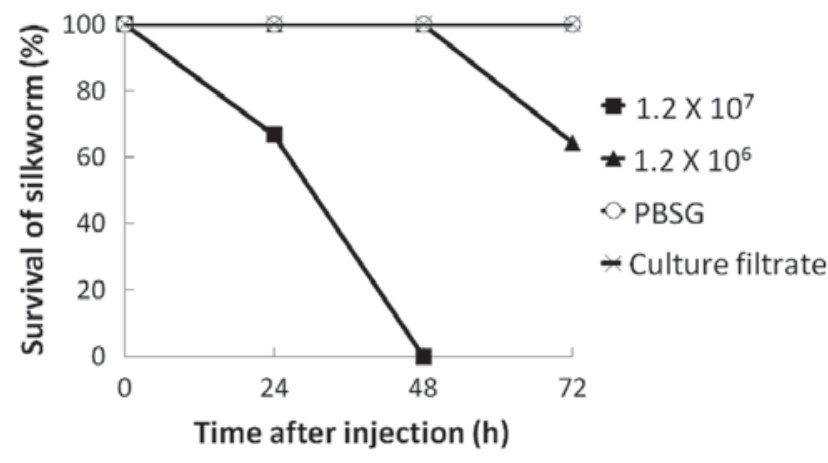

Figure 1. Lethality of $V$. vulnificus to silkworms. V. vulnificus OPU1-Rf bacterial cultures $\left(1.2 \times 10^{6}\right.$ or $1.2 \times 10^{7} \mathrm{cfu} /$ silkworm), V. vulnificus OPU1-Rf culture filtrates or PBSG were injected into the silkworm hemolymph. V. vulnificus, Vibrio vulnificus; PBSG, phosphate-buffered saline with $0.01 \%$ gelatin.

immediately following the injection, however, the animals behaved normally at $\sim 1 \mathrm{~h}$. This blunting of silkworm behavior was also observed when the animals were injected with the diluent (PBSG) alone. These findings may have been associated with the injection stimulus, an example being a decrease in body temperature caused by the injected solution. As shown in Fig. 1, the silkworms injected with $V$. vulnificus OPU1-Rf began to die at $24 \mathrm{~h}$. By contrast, the silkworms injected with PBSG or culture filtrate remained alive at $72 \mathrm{~h}$. In the deceased silkworms, the points of injection turned black in the initial hours, and these black spots gradually spread throughout the entire body. All silkworms injected with $1.2 \times 10^{7} \mathrm{cfu} / \mathrm{silkworm}$ died within $48 \mathrm{~h}$. A reduced dose of $1.2 \times 10^{6} \mathrm{cfu} / \mathrm{silkworm}$ extended survival rates, and $60 \%$ of the worms were alive at $72 \mathrm{~h}$ (Fig. 1). Thus, the virulence of $V$. vulnificus towards silkworms may be dose-dependent at these doses, although only two doses were examined in the present study. These results indicated that $V$. vulnificus is life-threatening to silkworms, and that silkworms can be used for investigating the virulence of $V$. vulnificus. As the injection of $1.2 \times 10^{7} \mathrm{cfu} / \mathrm{silkworm}$ led to the death of all silkworms within $48 \mathrm{~h}, 10^{7} \mathrm{cfu} /$ silkworm was used in the subsequent experiments.

Screening of the V. vulnificus-attenuated mutants through the assessment of silkworm lethality. As the silkworms were found to be sensitive to $V$. vulnificus infection, the present study attempted to use a silkworm infection model to identify the pathogenic genes of $V$. vulnificus against silkworms. The attenuated mutants, which had lost pathogenicity to silkworms, were screened from the 1,016 transposon insertion mutants.

The transposon insertion mutants were cultured for 4-6 h at $37^{\circ} \mathrm{C}$ with agitation. Culture fluid with an $\mathrm{OD}_{600}$ of 1.0 was diluted with PBSG, and a 50- $\mu 1$ aliquot was injected into the hemolymph of silkworms. Subsequently, $\sim 3.3 \times 10^{7} \pm 2.9 \times 10^{7} \mathrm{cfu}$ of the organism were injected into each silkworm, and silkworm status (dead or alive) was monitored for 5 days. In the process of identifying less virulent mutants, which did not lead to silkworm death within 5 days, 78 candidates were obtained (primary screening). To reduce experimental error, a second inoculation of the first candidate mutant was performed. Of the 78 candidates, 16 mutants did not lead to silkworm death within 5 days of injection (secondary screening). To confirm the avirulent properties of the secondary screened candidates,

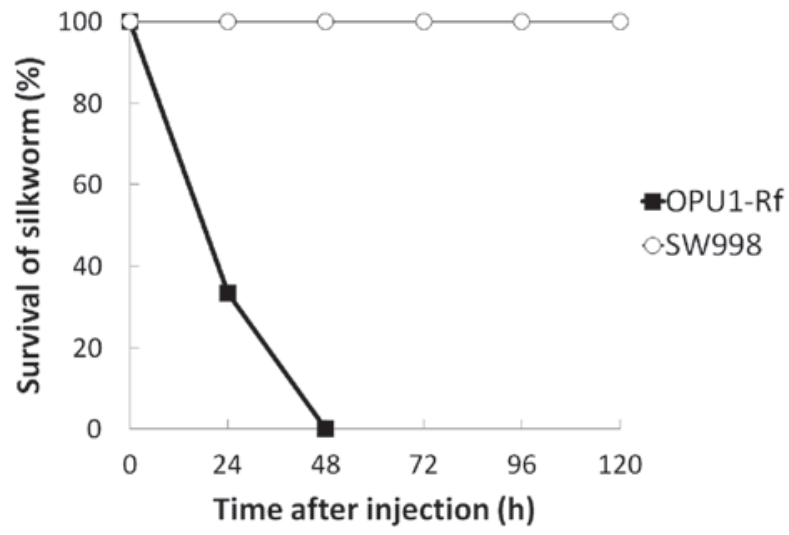

Figure 2. Survival durations of silkworms following being injected with V. vulnificus SW998. A total of five silkworms were injected, each with diluted culture media of the V. vulnificus OPU1-Rf parent strain and the transposon-inserted mutant SW998. V. vulnificus, Vibrio vulnificus; Rf, rifampicin-resistant OPU1 strain.

each of the 16 secondary screened candidates was injected into five silkworms. The candidates, in which all five injected silkworms survived for 5 days, were selected as attenuated mutants. Of the 16 mutants, 15 were not selected as an attenuated mutant as the silkworms died during the observation period. The SW998 mutant was found to be avirulent to all five silkworms (Fig. 2).

Transposon insertion sites in the attenuated mutant. As SW998 was avirulent in the silkworms (Fig. 2), the virulence-associated gene was expected to have been disrupted by the transposon insertion in SW998. In order to locate the transposon-inserted gene in the SW998 genome, recombinant plasmid DNA which contained the transposon insertion site was prepared as aforementioned and the DNA sequences adjacent to the transposon insertion sites were determined (Fig. 3). The nucleotide sequences of the cloned V. vulnificus DNA fragment adjacent to the transposon insertion site showed a high level of homology to the $V$. vulnificus rtxA gene, which has been reported as a $V$. vulnificus cytotoxic factor (24).

\section{Discussion}

Certain in vivo experiments designed to investigate the precise mechanisms of microbial infections are difficult to replace using in vitro experiments or models. Mice are often used to investigate $V$. vulnificus infection; however, using a larger number of animals may not always be economically or ethically feasible. Random transposon insertion mutagenesis and subsequent screening of attenuated mutants have been applied to identify virulence genes in several bacterial pathogens (25). Thousands of mice are required to isolate attenuated mutants. Animal experiments using mammals are required; however, to obtain satisfactory results, the sacrifice of a large number of animals may be required. In vivo infection models using invertebrates instead of mammals have been successfully applied in pathogenic microbe investigations, including Staphylococcus aureus virulence genes identified using a silkworm infection model (18). The purpose of the present study 

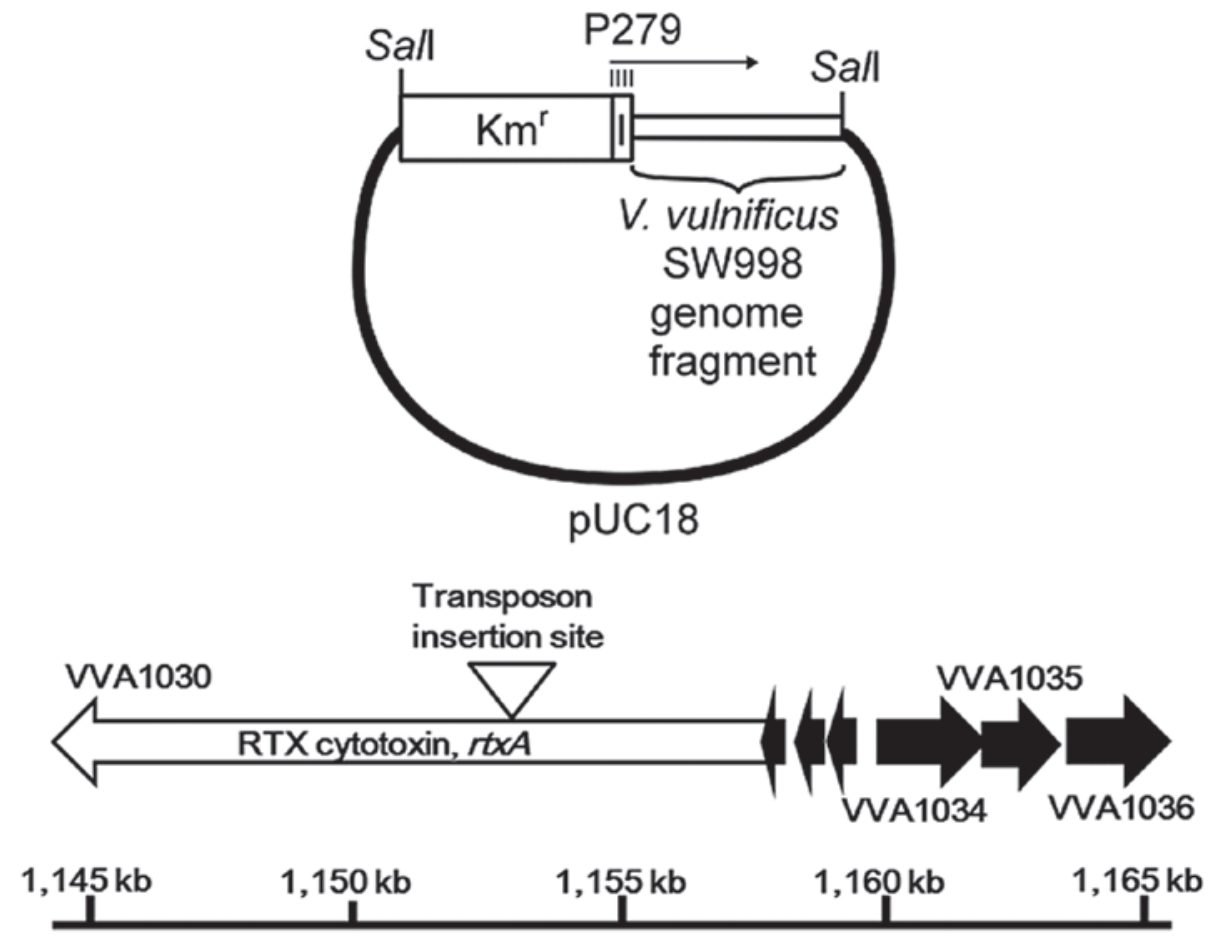

Figure 3. Transposon insertion site of V.vulnificus SW998. (A) To identify the transposon-inserted gene, the V. vulnificus SW998 transposon insertion region was cloned into the pUC18 plasmid vector, as described previously (18). The SalI-digested genomic DNA of $V$. vulnificus SW998 was cloned into the pUC18 SalI site with $\mathrm{Km}^{\mathrm{r}}$ as an indicator. The P279 oligonucleotide primer, which was designed to hybridize to the I-ends of the mini-Tn5Km2 transposon, was used to amplify the $V$. vulnificus SW998 transposon insertion site The arrow shows the direction of DNA sequencing. (B) Schematic representation of the $V$. vulnificus chromosomal region around the rtx element and transposon insertion site. Arrows indicate transcriptional directions and coding regions of $V$. vulnificus YJ016 genes (accession no. NC_005140). The line below indicates the gene location. V. vulnificus, Vibrio vulnificus; Km ${ }^{\mathrm{r}}$, kanamycin resistance gene.

was to examine whether the silkworm infection model can be applied to investigate the virulence of $V$. vulnificus.

In the present study, silkworms were demonstrated to be sensitive to the $V$. vulnificus; inoculation of this bacterium into the hemolymph of animals led to death (Fig. 1). Kaito et al (26) reported that several bacterial species, including $S$. aureus, Streptococcus pyogenes, Pseudomonas aeruginosa and $V$. cholerae, which are human pathogens, can also infect and induce death in silkworms when injected into the blood stream of silkworms, whereas non-pathogenic laboratory strains of E. coli cannot. Although there is no direct evidence to confirm whether the death of silkworms is caused by the same factors as mammals, silkworms may recognize virulence factors of pathogens as they have the Toll and immune deficiency pathways, which are homologous to the mammalian Toll-like receptor and tumor necrosis factor receptor signaling pathways, respectively (27). Using the silkworm infection model, the SW998 attenuated mutant strain was obtained in the present study, in which the $\operatorname{rtx} A$ gene was disrupted by a transposon insertion. The $r t x A$ gene is a member of the $r t x$ gene cluster in the $V$. vulnificus genome. RtxA has been suggested to be a toxin essential for $V$. vulnificus virulence, which has been confirmed by experimental infection of a mouse model and in an in vitro tissue culture model (24). In the present study, the injection of culture filtrate did not induce silkworm death (Fig. 1), which suggested that the expression of RtxA increased following interaction of the pathogen with host cells, as reported previously (28). Therefore, the bacteria secreted RtxA in the silkworm in vivo and RtxA may be toxic to silkworms. Complete removal of the genes from the genome and lethality assessment are required to determine whether RtxA is actually toxic to silkworms.

In conclusion, the silkworms examined in the present study died when inoculated experimentally with $V$. vulnificus and the $\operatorname{rtx} A$ gene was identified successfully using this infection model. It is expected this model may be useful for investigating $V$. vulnificus virulence factors and assist in developing effective therapies to protect against $V$. vulnificus infection.

\section{Acknowledgements}

The authors would like to thank Dr Toru Takahashi (Okayama Prefectural University, Okayama, Japan) for their advice during preparation of the manuscript. The present study was supported in part by JSPS Kakenhi (grant no. 22590400) and the Grant for Special Scientific Research of Okayama Prefectural University, 2012.

\section{References}

1. de Araujo MR, Aquino C, Scaramal E, Ciola CS, Schettino G and Machado MC: Vibrio vulnificus infection in São Paulo, Brazil: Case report and literature review. Braz J Infect Dis 11: 302-305, 2007.

2. Vinh DC, Mubareka S, Fatoye B, Plourde P and Orr P: Vibrio vulnificus septicemia after handling Tilapia species fish: A Canadian case report and review. Can J Infect Dis Med Microbiol 17: 129-132, 2006.

3. Horseman MA and Surani S: A comprehensive review of Vibrio vulnificus: An important cause of severe sepsis and skin and soft-tissue infection. Int J Infect Dis 15: e157-e166, 2011. 
4. Hlady WG and Klontz KC: The epidemiology of Vibrio infections in Florida, 1981-1993. J Infect Dis 173: 1176-1183, 1996.

5. Miyasaka J, Yahiro S, Arahira Y, Tokunaga H, Katsuki K and Hara-Kudo Y: Isolation of Vibrio parahaemolyticus and Vibrio vulnificus from wild aquatic birds in Japan. Epidemiol Infect 134: 780-785, 2006

6. Osaka K, Komatsuzaki M, Takahashi H, Sakano S and Okabe N Vibrio vulnificus septicaemia in Japan: An estimated number of infections and physicians' knowledge of the syndrome. Epidemiol Infect 132: 993-996, 2004.

7. National Enteric Disease Surveillance, Centers for Disease Control and Prevention, Atlanta, Georgia, USA: COVIS Annua Summary, 2012. www.cdc.gov/ncezid/dfwed/pdfs/covis-annualreport-2012-508c.pdf

8. Fouz B, Roig FJ and Amaro C: Phenotypic and genotypic characterization of a new fish-virulent Vibrio vulnificus serovar that lacks potential to infect humans. Microbiology 153: 1926-1934, 2007.

9. Bier N, Bechlars S, Diescher S, Klein F, Hauk G, Duty O, Strauch E and Dieckmann R: Genotypic diversity and virulence characteristics of clinical and environmental Vibrio vulnificus isolates from the Baltic Sea region. Appl Environ Microbiol 79: 3570-3581, 2013.

10. Kreger, A. and Lockwood, D: Detection of extracellular toxin(s) produced by Vibrio vulnificus. Infect. Immun. 33: 583-590, 1981.

11. Miyoshi S, Oh EG, Hirata K and Shinoda S: Exocellular toxic factors produced by Vibrio vulnificus. J Toxicol Rev 12: 253-288, 1993.

12. Powell JL, Wright AC, Wasserman, SS, Hone DM and Morris JG Jr: Release of tumor necrosis factor alpha in response to Vibrio vulnificus capsular polysaccharide in vivo and in vitro models. Infect Immun 65: 3713-3718, 1997.

13. Jeong HG, Oh MH, Kim BS, Lee MY, Han HJ, and Choi SH: The capability of catabolic utilization of $\mathrm{N}$-acetylneuraminic acid, a sialic acid, is essential for Vibrio vulnificus pathogenesis. Infect Immun 77: 3209-3217, 2009

14. Hor LI, Chang YK, Chang CC, Lei HY and Ou JT: Mechanism of high susceptibility of iron-overloaded mouse to Vibrio vulnificus infection. Microbiol Immunol 44: 871-878, 2000.

15. Bae T, Banger AK, Wallace A, Glass EM, Aslund F, Schneewind O and Missiakas DM: Staphylococcus aureus virulence genes identified by bursa aurealis mutagenesis and nematode killing. Proc Natl Acad Sci USA 101: 12312-12317, 2004.

16. Seed KD and Dennis JJ: Development of Galleria mellonella as an alternative infection model for the Burkholderia cepacia complex. Infect Immun 76: 1267-1275, 2008.
17. Mylonakis E and Aballay A: Worms and flies as genetically tractable animal models to study host-pathogen interactions. Infect Immun 73: 3833-3841, 2005.

18. Kaito C, Kurokawa K, Matsumoto Y, Terao Y, Kawabata S, Hamada S and Sekimizu K: Silkworm pathogenic bacteria infection model for identification of novel virulence genes. Mol Microbiol 56: 934-944, 2005.

19. Metcalf WW, Jiang W and Wanner BL: Use of the rep technique for allele replacement to construct new Escherichia coli hosts for maintenance of R6K gamma origin plasmids at different copy numbers. Gene 138: 1-7, 1994.

20. Hensel M, Shea JE, Gleeson C, Jones MD, Dalton E and Holden DW: Simultaneous identification of bacterial virulence genes by negative selection. Science 269: 400-403, 1995.

21. Sambrook J and Russell DW: Molecular Cloning: A laboratory manual. Cold Spring Harbor Laboratory Press, Cold Spring Harbor, New York, Vol. 3, Appendix 2.2, 2001

22. Hamamoto H, Kurokawa K, Kaito C, Kamura K, Manitra Razanajatovo I, Kusuhara H, Santa T and Sekimizu K: Quantitative evaluation of the therapeutic effects of antibiotics using silkworms infected with human pathogenic microorganisms. Antimicrob Agents Chemother 48: 774-779, 2004.

23. Yamamoto $\mathrm{M}$, Kashimoto T, Tong $\mathrm{P}$, Xiao J, Sugiyama $\mathrm{M}$, Inoue M, Matsunaga R, Hosohara K, Nakata K, Yokota K, et al: Signature-tagged mutagenesis of Vibrio vulnificus. J Vet Med Sci 77: 823-828, 2015.

24. Lee JH, Kim MW, Kim BS, Kim SM, Lee BC, Kim TS and Choi SH: Identification and characterization of the Vibrio vulnificus rtxA essential for cytotoxicity in vitro and virulence in mice. J Microbiol 45: 146-152, 2007.

25. Saenz HL and Dehio C: Signature-tagged mutagenesis: Technical advances in a negative selection method for virulence gene identification. Curr Opin Microbiol 8: 612-619, 2005.

26. Kaito C, Akimitsu N, Watanabe H and Sekimizu K: Silkworm larvae as an animal model of bacterial infection pathogenic to humans. Microb Pathog 32: 183-190, 2002.

27. Tanaka $\mathrm{H}$ and Yamakawa $\mathrm{M}$ : Regulation of the innate immune responses in the silkworm, Bombyx mori. ISJ 8: 59-69, 2011.

28. Kim YR, Lee SE, Kook H, Yeom JA, Na HS, Kim SY, Chung SS, Choy HE and Rhee JH: Vibrio vulnificus RTX toxin kills host cells only after contact of the bacteria with host cells. Cell Microbiol 10: 848-862, 2008. 